\title{
In Situ Fenton Triggered PDA Coating Copper Mesh with Underwater Superoleophobic Property for Oily Wastewater Pretreatment
}

\author{
Ying $X u^{1,2, *}$, Wei Wang ${ }^{3}$, Zhigao Zhu ${ }^{4}$ and Bin $X u^{1, *}$ \\ 1 Yangtze River Scientific Research Institute of Changjiang Water Resources Commission, Wuhan 430010, China \\ 2 School of Ecology and Environment, Zhengzhou University, Zhengzhou 450001, China \\ 3 State Key Laboratory of Urban Water Resource and Environment, Harbin Institute of Technology, \\ Harbin 150001, China; wangweirs@hit.edu.cn \\ 4 Key Laboratory of New Membrane Materials, Ministry of Industry and Information Technology, \\ School of Environment and Biological Engineering, Nanjing University of Science and Technology, \\ Nanjing 210094, China; zhigaozhu@outlook.com \\ * Correspondence: xuying@zzu.edu.cn (Y.X.); taibaidugu@163.com (B.X.); Tel.: +86-1311-560-1205 (Y.X. \& B.X.)
}

check for updates

Citation: Xu, Y.; Wang, W.; Zhu, Z.; $X u, B$. In Situ Fenton Triggered PDA Coating Copper Mesh with Underwater Superoleophobic Property for Oily Wastewater Pretreatment. Processes 2021, 9, 1665. https://doi.org/10.3390/pr9091665

Academic Editor: Chuanyi Wang

Received: 7 August 2021

Accepted: 8 September 2021

Published: 15 September 2021

Publisher's Note: MDPI stays neutral with regard to jurisdictional claims in published maps and institutional affiliations.

Copyright: (c) 2021 by the authors. Licensee MDPI, Basel, Switzerland. This article is an open access article distributed under the terms and conditions of the Creative Commons Attribution (CC BY) license (https:// creativecommons.org/licenses/by/ $4.0 /)$.

\begin{abstract}
The issue of oily wastewater treatment has become a worldwide challenge due to increasing industrial oily wastewater and frequent oil spill accidents. As an integral part of practical sewage treatment, pretreatment is conducted to remove inorganic particles, floating oil, and some emulsified oil, and to pave the way for post-treatment. Here, we report a facile fabricated, hydrostable, and rapid underwater-formed superoleophobic copper mesh with polydopamine (PDA) coating for efficient oily wastewater pre-treatment. Unlike with traditional technologies, using the interface phenomenon to solve the problem of oil/water mixture separation provided a new approach for the low energy input pretreatment process. The PDA coating formed by the in situ Fenton method not only rapidly constructs a protection layer for the etched hierarchical micro-size particles on mesh and results in enhanced hydrophilicity, but also exhibits high uniformity and enhanced stability in acid/alkali medium. Benefiting from the above processes, a very high flux of $25 \mathrm{~L} \mathrm{~m}^{-2} \mathrm{~s}^{-1}$ and high separation efficiency of $99.0 \%$ toward various oil/water mixtures were achieved, revealing excellent prospects for practical usage. Therefore, this new approach offered insight into the development of a cost-effective and functional method for efficient pretreatment of oily wastewater.
\end{abstract}

Keywords: in situ Fenton; polydopamine; underwater superoleophobic; oily wastewater pretreatment

\section{Introduction}

The increasing amount of oily wastewater discharged from daily life and industrial processes, such as cooking waste, steel, aluminum, food, textile, leather, and petrochemical metal production, threatens not only the aquatic ecosystem but also human health $[1,2]$. Oily sewage generally consists of inorganic particles, floating oil, emulsified oil, and soluble oil, which require multiple processes to deal with $[3,4]$. As an integral part of practical engineering application, pre-treatment is conducted to remove inorganic particles, floating oil, and some emulsified oil, paving the way for post-treatments [5]. Conventional oily wastewater pretreatment process methods such as coagulation-flocculation, skimming, and centrifugation are limited by low separation efficiency, high energy cost, and tedious manual operation [6]. Hence, a new pretreatment approach with strong machine performance for inorganic particle interception, good oil/water separation capability for large flux pretreatment, and low energy input is highly desired.

The invention of superwetting surfaces opens up new insights and routes for the key part of oily sewage pretreatment: oil/water mixture separation [7,8]. Different from the energy-consuming process of traditional technology, super-wetting material can separate oil and water through interfacial action under the condition of only using gravity; hence, the 
operating cost is much lower $[9,10]$. As a typical example, inspired by the phenomenon that fish can keep their surfaces clean in oil-polluted water, Jiang and co-workers first developed an alternative method of preparing an underwater superoleophobic interface in an aqueous medium [11]. Even though underwater superoleophobic materials have been intensively investigated, they still suffer from high cost, multistep or unstable problems, and are unable to adapt to the complex environment required for pre-processing [12,13]. Thus, the development of robust, hydrostable, and underwater superoleophobic material via an easy and cost-effective method, for the pretreatment stage in particular, remains challenging.

Metallic meshes with high mechanical strength, large-scale production, and high filtration flux have been widely studied for oil/water separation [14]. To date, various methods, including the sol-gel method, hydrothermal methods, and chemical vapor deposition, have been employed to construct the roughness of substrate [15-17]. Therein, the etching process has been considered one of the simplest routes in metal surface treatment, as it can directly obtain rough structure without changing surface composition. However, the etching process is conducted in an intense corrosion environment, which could directly destroy the protective layer of the metallic meshes, resulting in a fragile surface. The destroyed structure of a mesh cannot be operated for long-term running in practical applications. Thus, it is envisioned that a hierarchical structure-based underwater superhydrophobic copper mesh would be endowed with a stable feature to maintain high separation performance and avoid direct corrosion in long-term running.

Dopamine (DA), a biometric material with adhesive proteins and unique wet adhesion in marine mussels, could be self-polymerized and form a polydopamine (PDA) coating layer with hydrophilicity and excellent biocompatibility for various substrates $[18,19]$. However, it has previously been reported that PDA-based substrates are unstable in acid and alkaline solution due to the non-covalent value of the PDA layer and gradually wear off in an agitated aqueous environment [20,21]. Most importantly, the polymerization process is time-consuming. It was then reported that the polymerization of PDA could be triggered by Fenton's reagents, which exhibited a fast deposition rate, uniform coating layer, and strong acid/alkali resistance [22-24]. Hence, if a PDA coating layer can be attached to the etched copper mesh, the leaking copper ions will cooperate with the polymerization of the dopamine, and the corrosion of the mesh and reaction time of PDA would be both reduced.

In this work, we rationally designed an underwater superoleophobic mesh by the combination of the etching method and in situ Fenton-triggered PDA coating layer. The microstructures in combination with the hydrophilic groups of the PDA layer endowed the mesh with robust mechanical strength for inorganic particles, high separation efficiency, and ultrahigh water flux for oil/water mixture, anticorrosion, and excellent reusability. Herein, we anticipate that this easily scaled-up, facile fabricated, well oil/water mixture separated, inorganic-particle-resistant underwater superoleophobic copper mesh holds great potential in future oily wastewater preprocessing.

\section{Materials and Methods}

\subsection{Materials}

Dopamine hydrochloride was purchased from Aladdin Industrial Inc., Shanghai, China. Anhydrous ethanol, ferric chloride, hydrochloric acid, methylene blue, Sudan Red IV, and oil (chloroform, 1,2-dichloroethane, and hexane) were obtained from China National Medicines Corporation Ltd. Copper mesh, sandpaper, and rapeseed oil were purchased from a local store. Chemical reagents were of analytical grade and used without further purification.

\subsection{Fabrication of PDA-Coated Copper Mesh}

First, a piece of copper mesh $(3 \times 3 \mathrm{~cm})$ was ultrasonically rinsed in turn with deionized water and ethanol at least 3 times, and then dried with nitrogen flow. Then, $1.625 \mathrm{~g}$ of ferric chloride was dissolved in a $100 \mathrm{~mL} \mathrm{HCl}$ solution $(0.1 \mathrm{M})$. Subsequently, the copper mesh was immersed in the above solution various times to achieve optimum surface struc- 
ture. The copper meshes etched by $\mathrm{FeCl}_{3}$ for 10, 20, and 30 min were named as $\mathrm{ETCu} / 10$, $\mathrm{ETCu} / 20$, and ETCu/30, respectively. Second, the etched meshes were immersed in $100 \mathrm{~mL}$ of tris(hydroxyethyl)aminomethane solution $(10 \mathrm{mM}, \mathrm{pH}=8.5)$. Then, $200 \mathrm{mg}$ of dopamine and $19.6 \mathrm{mH}$ of $\mathrm{H}_{2} \mathrm{O}_{2}$ were added to the above solution simultaneously, followed by stirring at ambient temperature for 20, 40, and $60 \mathrm{~min}$, and named as PDA20/ETCu, PDA40/ETCu, and PDA60/ETCu, respectively. In contrast, $200 \mathrm{mg}$ of dopamine was added to the mixture without $\mathrm{H}_{2} \mathrm{O}_{2}$ at ambient temperature for $24 \mathrm{~h}$ and named PDA/ETCu. The meshes were washed with deionized water and dried in an oven at $40{ }^{\circ} \mathrm{C}$ for $6 \mathrm{~h}$.

\subsection{Oil/Water Seperation}

A mixture of oil dyed with Sudan Red IV and water dyed with methylene blue (40\% $v / v$ ) was poured slowly into a filtering system. The filter mesh was placed in the middle of the separation device with appropriate seals. Four types of oils, including rapeseed oil, toluene, gasoline, and hexane, were used and the oils were mixed with water. The $0.1 \mathrm{~L}$ oil/water mixture was poured into the filtering system ten times to test the reusability of the copper mesh. We predict that the PDA-coated copper mesh can be applied in the preprocessing tank of raw oily wastewater as shown in Figure 1.

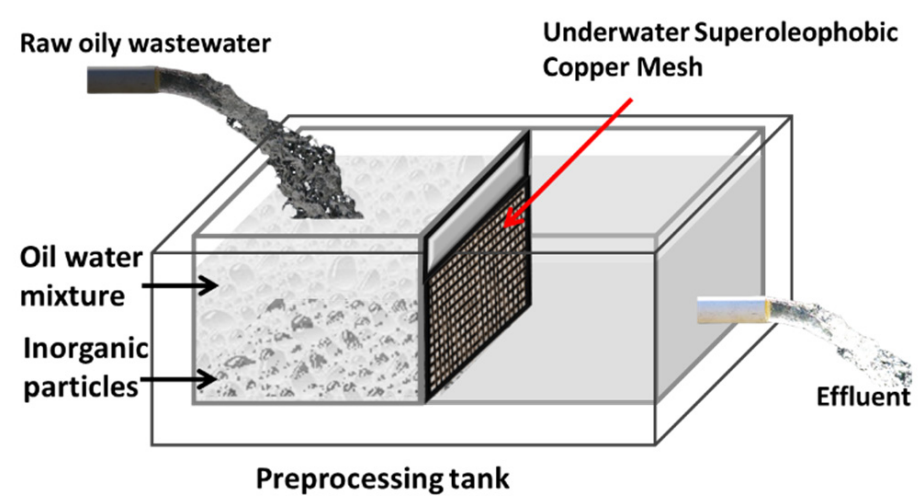

Figure 1. The schematic illustration of the application of copper mesh in a preprocessing tank of raw oily wastewater.

\subsection{Mechanical Durability and Chemical Stability}

Light oil (take hexane as an example) and corrosive solution ( $\mathrm{HCl}$ solution, $\mathrm{pH}=3$; $\mathrm{NaOH}$ solution, $\mathrm{pH}=10$; and $1 \mathrm{M} \mathrm{NaCl}$, solution $\mathrm{pH}=7$ ) mixtures were prepared several times to examine the chemical stability of the mesh in oil/water separation. To further confirm the chemical stability of PDA and release behavior of $\mathrm{Cu}$ ions from the mesh, the $3 \times 3 \mathrm{~cm}$ meshes were incubated in $50 \mathrm{~mL}$ of corrosive solution in a small glass vial for $12 \mathrm{~h}$. Then, the solutions were tested by UV/Vis spectrophotometry (UV2550, Shimadzu, Kyoto, Japan) for PDA release, and by inductively coupled plasma analysis (ICP, Optima 5300 CV, Perkin Elmer Inc., Shanghai, China) for the study of $\mathrm{Cu}$ ion release.

As for the abrasion test, sandpaper was used to simulate the friction circumstances. A $200 \mathrm{~g}$ stainless steel weight was put on the copper mesh to increase the load. To avoid uneven loading, a piece of glass was put between the weight and mesh, and the mesh was glued on the glass using twin adhesive. The sample was moved $5 \mathrm{~cm}$ with a speed of $0.5 \mathrm{~cm} / \mathrm{s}$ on the sandpaper (abrasive paper, $100 \mathrm{mesh}$ ).

\subsection{Characterizations}

The images were obtained by a field emission scanning electron microscope (SEM, Microlab600i, 2.0 kV, Zeiss, Oberkochen, Germany). X-ray photoelectron spectroscopy (XPS) measurements were performed using a Thermo ESCALAB 250 with Al K $\alpha$ X-ray $(\mathrm{h} v=1486.6 \mathrm{eV})$ radiation. Water contact angles (WCAs) and oil contact angles (OCAs) of $2 \mu \mathrm{L}$ were performed using a contact angle goniometer (SL200B, Kino, Shanghai, China). 
The average value of three measurements performed at different positions on the same sample was adopted as the contact angle.

\section{Results and Discussion}

\subsection{Fabrication and Characterizations of the Samples}

The introduction of the hierarchical structure via the etching process is expected to form a nano/micro scale roughness on copper mesh, thus inducing superhydrophilicity and underwater superoleophobicity. The schematic description of the preparation of PDAcoated copper mesh is shown in Figure 2a. The morphologies of copper mesh etched at each stage were typically monitored by SEM. The pristine copper mesh exhibited neat rule arrangement with an average pore size of $40 \mu \mathrm{m}$ ( 400 mesh size, Figure $2 \mathrm{~b})$, revealing a smooth surface without any complex structures. The surface roughness was dependent on the etching time. With the increasing etching time, the ETCu20 surface exhibited uniform and compact structures with a domain-like rough microstructure (Figure 2c), which closely resembled the lotus leaf and fish-scale surfaces. In contrast, there was no obvious difference on the ETCu10 surface (Figure S1a) due to the $\mathrm{CuO}$ protective layer, and the roughness of the ETCu/30 surface structure was enhanced (Figure S1 band c) due to the serious damage caused by the harsh corrosion solution. To guarantee the mechanical strength and efficient use of time and materials, ETCu/20 was chosen as the optimal condition for the following experiments.

(a)
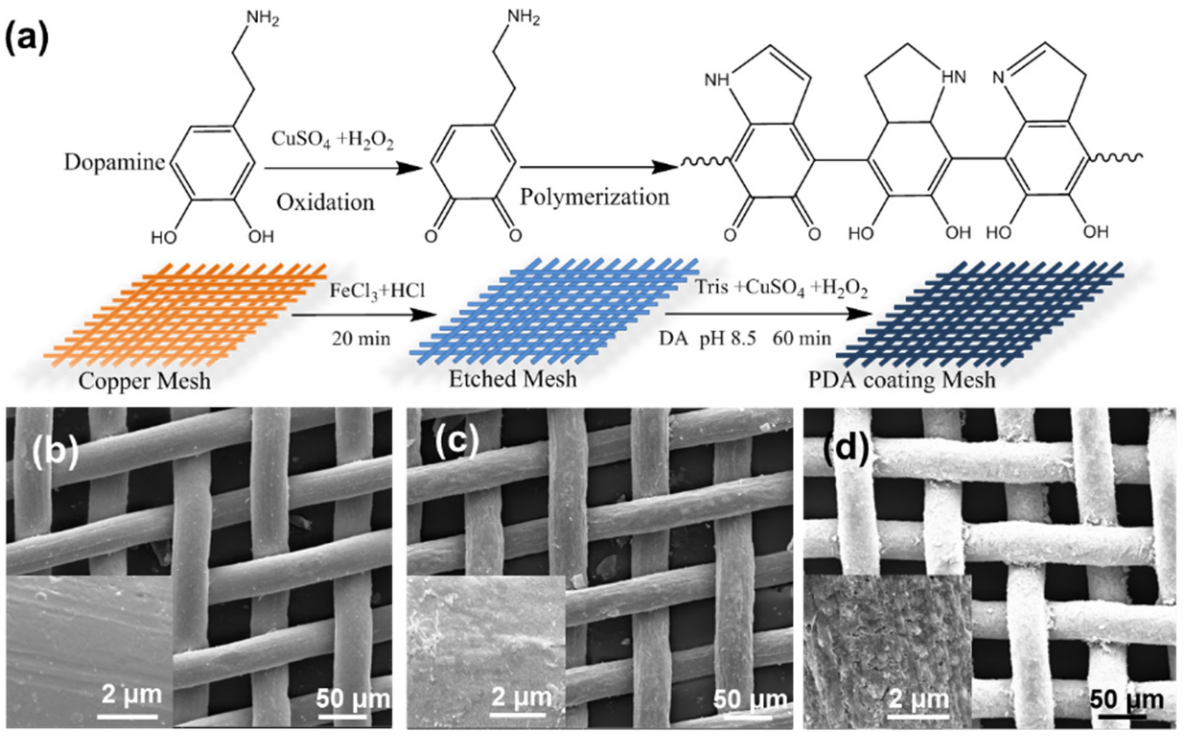

Figure 2. (a) Schematic description of the preparation of PDA-coated copper mesh. (b) SEM images of pristine copper mesh, (c) the etched mesh within $20 \mathrm{~min}$ and (d) the PDA decorated mesh within $60 \mathrm{~min}$.

The wettability of the mesh was exhaustively transformed from a hydrophobicity of $122^{\circ}$ to hydrophilicity of $0^{\circ}$ after the etching process as shown in Figure S2. To obtain the superhydrophilic and underwater superoleophobic property, the ETCu/20 was then coated with PDA, which not only enhanced the hydrophilic property of the hierarchical mesh but also protected the microstructures under terrible conditions. The morphologies and underwater superoleophobic properties were closely related to the deposition time, which was also systematically studied, as shown in Figure S3. The PDA formed in situ after $20 \mathrm{~min}$ and $40 \mathrm{~min}$ on the mesh exhibited an obviously thinner and more fragile layer compared to the $60 \mathrm{~min}$ deposition (Figure 2d); the underwater oil contact angles (UOCAs) of these copper meshes were $140.5 \pm 1.0^{\circ}, 149.2 \pm 1.0^{\circ}$, and $152.6 \pm 1.0^{\circ}$, respectively (Figure S4). Taking SEM images into consideration, the etched copper mesh coated with 
PDA after 60 min exhibited adequate thickness with underwater superoleophobic property, which was chosen as the optimum selection for the following oil/water separation.

The chemical composition of ETCu/20 and PDA-coated mesh (defined as PDA60/ETCu) was measured by XPS to further confirm the successful modification of PDA. As shown in Figure 3a, the peaks of PDA60/ETCu exhibited higher resolutions than those of ETCu/20, with a C1s peak of 284.9 , N1s peak of 400.1 , and O1s peak of $532.7 \mathrm{eV}$. Figure $3 \mathrm{~b}$ illustrates the increase of $\mathrm{N}$ content after dopamine self-polymerization on the mesh in detail, which was consistent with previous reports [20,21]. Moreover, for ETCu/20, the C1s signal can be curve-fitted to three peaks at the binding energies of $284.8,286.0$, and $289.0 \mathrm{eV}$, corresponding to the $\mathrm{C}-\mathrm{C} / \mathrm{C}-\mathrm{H}, \mathrm{C}=\mathrm{O}$, and $\mathrm{O}-\mathrm{C}=\mathrm{O}$ species, respectively (Figure $3 \mathrm{c}$ ). Subsequently, the $\mathrm{C}-\mathrm{N}$ species at $286.2 \mathrm{eV}$ appeared after PDA coating for about $60 \mathrm{~min}$, and this peak, associated with $\mathrm{C}-\mathrm{C} / \mathrm{C}-\mathrm{H}$ and $\mathrm{C}=\mathrm{O}$, became dominant (Figure $3 \mathrm{~d}$ ).
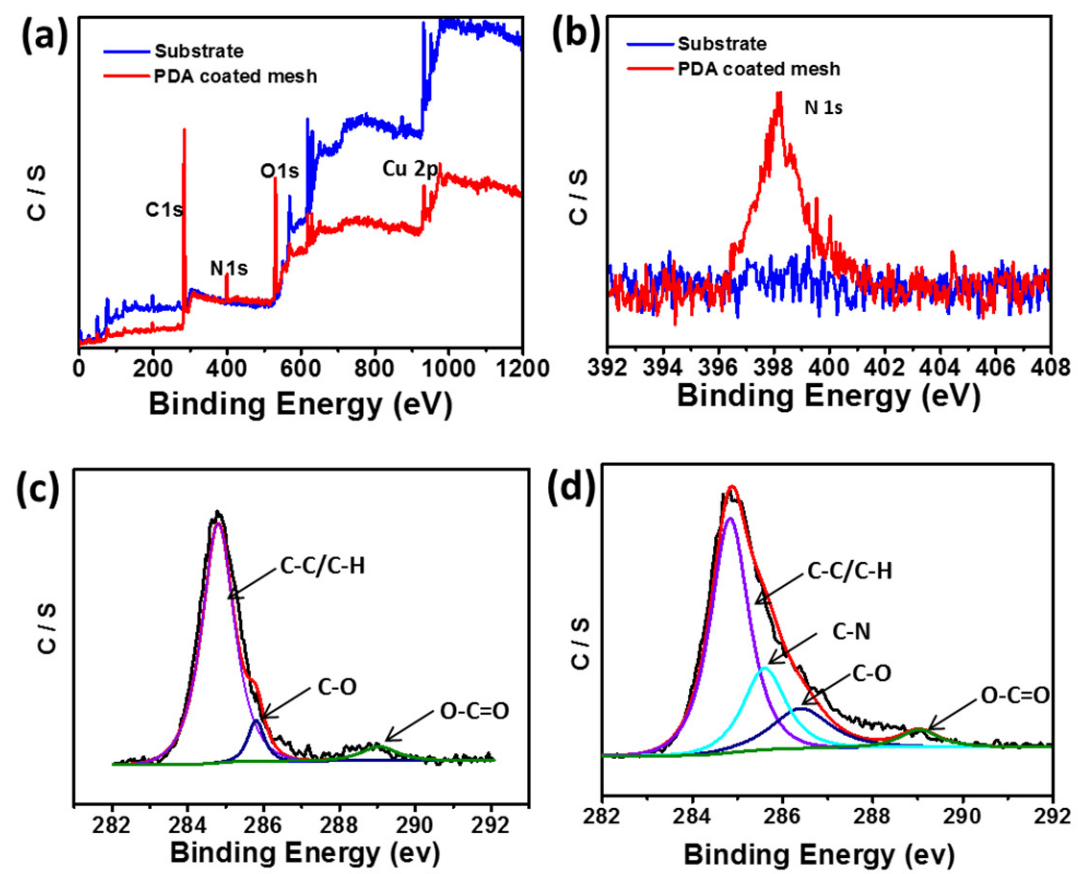

Figure 3. XPS spectra of the as-prepared mesh: (a) Survey scans of the spectral region from 0 to $1200 \mathrm{eV}$. (b) High-resolution XPS N1s narrow scans as a function of electron binding energy (BE). High-resolution XPS spectra of C1s peaks for (c) ETCu/20 and (d) PDA60/ETCu.

\subsection{Wettablity and Oil/Water Seperation}

The surface wettability performance of the as-prepared PDA60/ETCu mesh was characterized by the water contact angle analyzer. As proven in Figure 4a-c, PDA60/ETCu exhibited a superhydrophilic in air with the WCA of $0^{\circ}$, and superoleophobicity underwater with the UOCA of $152^{\circ}$. This phenomenon could be attributed to the significantly higher adhesion work of water than that of oil according to Young-Dupré's equation [25]. To further investigate the dynamical wetting behavior of water on the meshes, an oil droplet was used to detect the adhesion process. As shown in Figure 4d, when the oil droplet was forced to sufficiently contact the mesh with a distinct compression, the oil could be easily lifted without obvious deformation, illustrating the extremely low oil adhesion force. To assess the quality of separation more quantitatively, oil/water mixtures were prepared by mixing rapeseed oil, toluene, gasoline, and hexane with water, respectively, to assess the quality of separation performance. Since the densities of the light oils in this case are lighter than water, the oils stayed above the water (Figure 4e). Upon the oil/water mixture touching the mesh, the water could quickly pass through the meshes and reach the conical flask. Meanwhile, all of the oils were retained above the mesh due to the underwater superoleophobic property. 


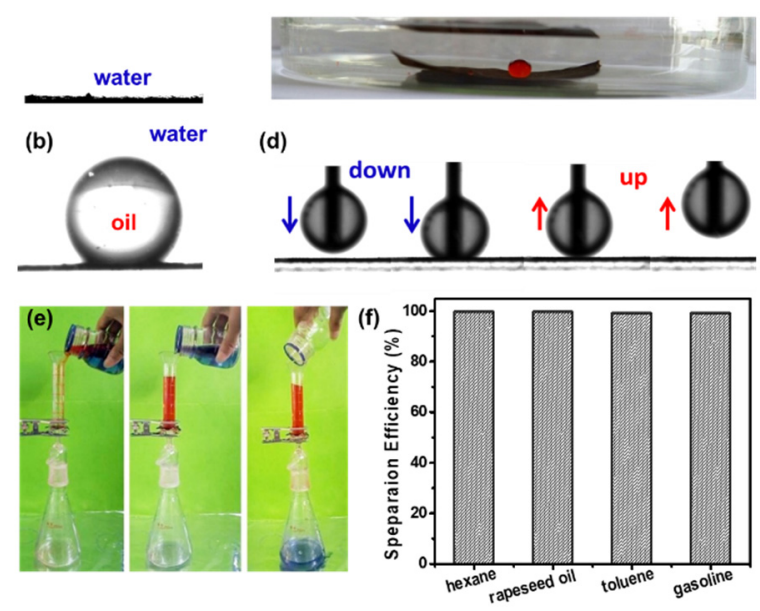

Figure 4. $(\mathbf{a}, \mathbf{b})$ The wetting behavior of the as-prepared mesh towards water in air and oil in water. (c) Photograph of the oil droplet on the coated mesh in water. (d) Photographs of the dynamic underwater oil-adhesion measurements on the coated mesh. (e) Photographs of the mixture of hexane and water being poured into the upper glass tube and separating well. (f) The separation efficiency of the mesh for different kinds of oils mixed water.

The separation efficiency was calculated by the oil rejection coefficient $\eta(\%)$ according to following equation:

$$
\eta=V_{\mathrm{p}} / \mathrm{V}_{0} \times 100
$$

where $V_{0}$ and $V_{P}$ are the oil volume of the original oil/water mixtures and the collected oil after the first separation, respectively. Moreover, the water flux (F) was also measured and calculated using the following equation:

$$
\mathrm{F}=\mathrm{V} /(\mathrm{S} \times \mathrm{t})
$$

where $\mathrm{V}$ is the volume of water that permeates through the membrane, $\mathrm{S}$ is the area of the mesh, and $t$ is the required time for the permeation. Here, we fixed $\mathrm{V}$ to $0.1 \mathrm{~L}$, and $\mathrm{t}$ is the required time for the permeation of $0.1 \mathrm{~L}$ of liquid. As for the separation process, the separation efficiencies of the series of oils were calculated to be above $99.0 \%$ (ranging from $99.6 \%$ to $99.0 \%$ ), as shown in Figure $4 \mathrm{f}$. The experimental results show that the water flux was $25 \mathrm{~L} \mathrm{~m}^{-2} \mathrm{~s}^{-1}$. In addition, the oil/water separation efficiency versus the recycle numbers was also investigated by taking the hexane-water mixture as an example. Ten cycles of separation tests were conducted, and the separation efficiency was calculated and recorded. The underwater superoleophobic mesh exhibited great performance in oil/water separation, which maintained a high separation efficiency of more than $99.0 \%$. Hence, our composite meshes are suitable for a variety of oil/water mixture separation due to their stability.

\subsection{Mechanical Durability and Corrosion Resistant Test}

In the pretreatment process, inorganic particles will produce certain wear on the film; therefore, the mechanical strength of the film and its coating should be guaranteed. The abrasive resistance of the coating layer on copper meshes determines the availability and economy of the separation membrane. Herein, to further test the durability of the as-prepared meshes, we used sandpaper to simulate the practical friction circumstances. The experimental setups are shown in Figure 5a,b. After abrasion, the surfaces of the meshes were destroyed a little, as shown in the SEM (Figure 5c). Despite the wear of the coating under strength damage, the main body of the copper mesh remained intact. Moreover, the UOCA remained above $150^{\circ}$, meaning that even though it is damaged, the mesh can still separate the oil/water mixture well. 

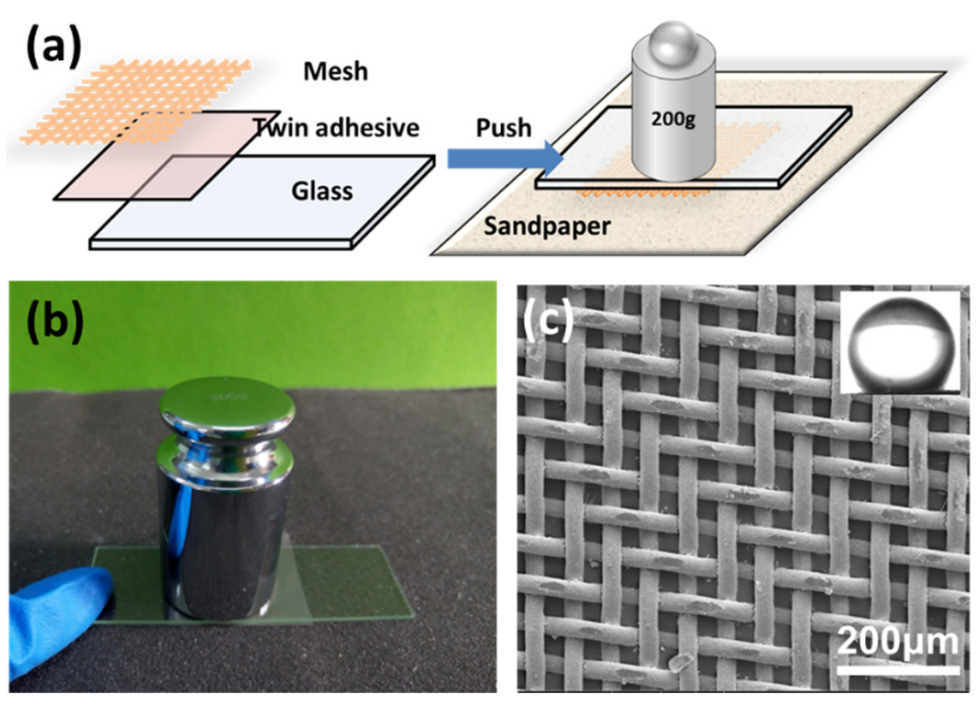

Figure 5. (a) Illustration of the abrasion test for the as-prepared copper mesh. (b) Photograph of the sandpaper abrasion test. (c) SEM of copper mesh after sandpaper abrasion test (inset: contact angle test).

Nanoparticles including inorganic or metal oxide are widely used to construct the hierarchical structure to improve the superwetting performance, but the leakage of nanoparticles or metal ions into the waterbody could cause secondary pollution. Hence, hydrostability is regarded as a crucial property of materials in long-term operation, especially in water treatment. Depositing the PDA layer on the etched mesh by the traditional polymerization method was also tested as a contrast. As shown in Figure S5a,b, the solution triggered via the Fenton method after 60 min exhibited a dark color, while the solution directly oxidized in air exhibited a transparent color. The copper ions in the polymerization process of PDA acted as cross-link sites by chelation with the amine and imine groups of PDA, thus accelerating the polymerization rate and improving the stability of the PDA coatings dramatically (Figure 6a).

(a)

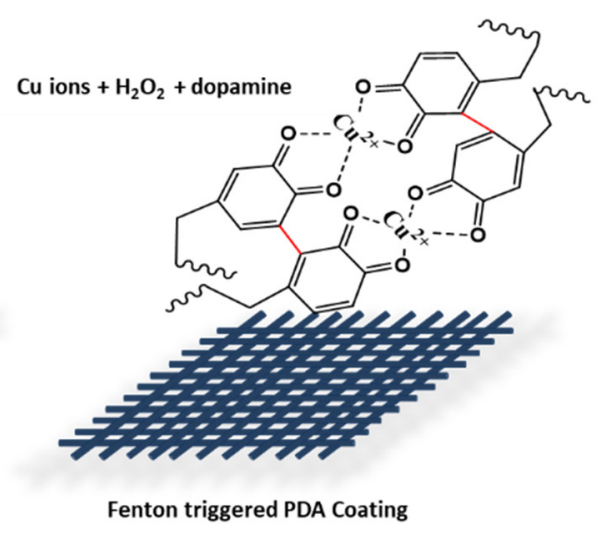

(b)

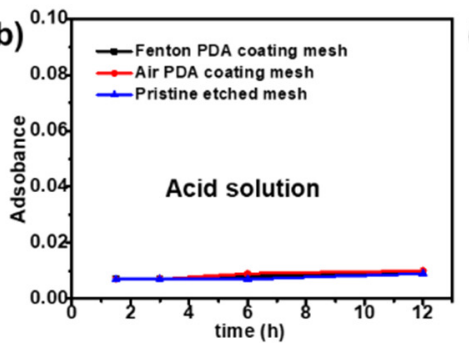

(d)

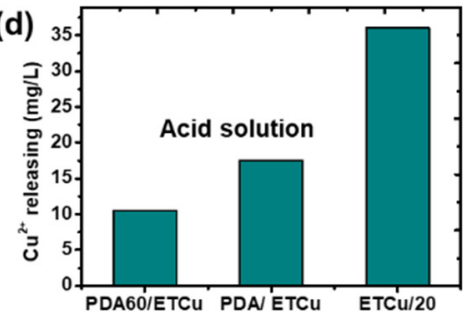

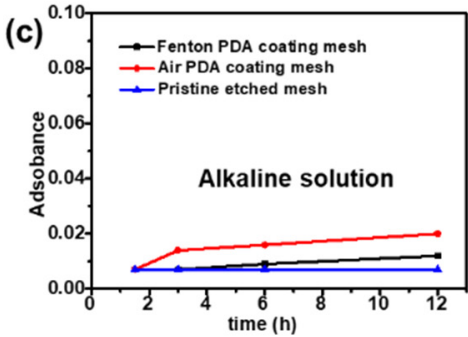

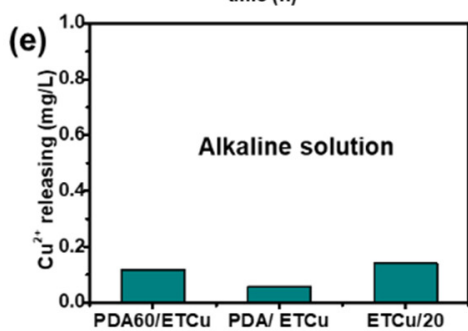

Figure 6. (a) The description of $\mathrm{Cu}$ ions participating in the polymerization of PDA layers on surfaces. The UV/Vis spectra of relevant copper mesh after immersion in (b) acid and (c) alkaline solution for $12 \mathrm{~h}$. The copper ions releasing from relevant copper mesh in (d) acid and (e) alkaline solution.

Simultaneously, the PDA layer formed using the traditional method exhibited irregular thickness after $24 \mathrm{~h}$, and parts of the mesh were still exposed to the air, thus corroding the mesh easily after the long running time (Figure S5c,d). Then, the air-oxidized PDA/ETCu, 
Fenton-triggered PDA60/ETCu, and ETCu/20 were immersed in acid/alkaline solution for $12 \mathrm{~h}$ to investigate the stability of the PDA-coating protective layer. UV/Vis spectrophotometry was used to measure the PDA degradation after immersion in corrosion solutions. As shown in Figure $6 \mathrm{~b}, \mathrm{c}$, PDA triggered via the Fenton method showed excellent stability on the copper mesh. The release of copper ions from the modified meshes PDA/ETCu, $\mathrm{PDA} 60 / \mathrm{ETCu}$, and ETCu $/ 20$ was also evaluated by ICP. The release contents of the relevant PDA-coated copper meshes (Figure 6d) were 10.57, 17.57, and $36.02 \mathrm{mg} / \mathrm{L}$, respectively, revealing that the in situ PDA coating can act as a protector of the mesh. The PDA60/ETCu not only exhibited a time saving of $60 \mathrm{~min}$, but also had better protective behavior than $\mathrm{PDA} / \mathrm{ETCu}$. Interestingly, the release rate of copper-based mesh in the acid solution was far more than in the alkaline solution due to the $\mathrm{CuO}$ layer formed after etching (Figure $6 \mathrm{~d}, \mathrm{e})$, revealing the strong antioxidant property of PDA trigged by the Fenton method.

\section{Conclusions}

In summary, we have presented an extremely facile, economical, and universal approach by in situ coating Fenton-triggered PDA on the etched copper mesh with strong robustness, hydro-stability, anticorrosion, and underwater superoleophobicity for efficient pretreatment of oily wastewater. It took an extremely short time for PDA to aggregate and coat on the mesh with adequate thickness and uniform homogeneity for constructing a protecting layer. The as-prepared composite copper mesh showed superhydrophilicity with WCA of $0^{\circ}$ and underwater superoleophobicity with OCA of $152^{\circ}$ for achieving efficient oil/water mixture separation. The aperture in the copper mesh guaranteed a large flux of $25 \mathrm{~L} \mathrm{~m}^{-2} \mathrm{~s}^{-1}$ driven only by gravity. More importantly, the as-prepared meshes showed enhanced stability and more durable underwater superoleophobicity toward various oil/water mixtures, even after 10 cycles, illustrating an excellent reusability. Great mechanical properties can effectively resist the destruction of inorganic particles in raw wastewater. Given the simplicity and versatility of this approach, combined with the low cost and protecting properties of copper mesh, this strategy provides new insight into design and development for long-term practical engineering applications.

Supplementary Materials: The following are available online at https: / www.mdpi.com/article / 10.3390/pr9091665/s1: Figure S1: SEM images of copper mesh etching with different time; Figure S2: The water contact angles of the pristine mesh and ETCu/20; Figure S3: SEM images of copper meshes coated by PDA with different time; Figure S4: The UOCAs of the copper meshes coated by Fenton-triggered PDA with different time; Figure S5: (a) Picture of 60 min PDA solution using Fenton-triggered PDA method. (b) Picture of $60 \mathrm{~min}$ PDA solution using traditional method. (c,d) SEM images of PDA coating using traditional method within $24 \mathrm{~h}$.

Author Contributions: Conceptualization, Y.X. and B.X.; data curation, Y.X.; writing-original draft preparation, Y.X.; writing-review and editing, Z.Z.; supervision, W.W. All authors have read and agreed to the published version of the manuscript.

Funding: Please add: This research was funded by National Natural Science Foundation of China (No. 52000161, 52000105, 51809009, and 52070052), the 68th batch of China Postdoctoral Science Foundation (No. 2020M682356), and the CRSRI Open Research Program (Program SN:CKWV2021892/KY).

Institutional Review Board Statement: Not applicable.

Informed Consent Statement: Not applicable.

Conflicts of Interest: The authors declare no conflict of interest.

\section{References}

1. You, Z.; Zhang, L.; Zhang, S.; Sun, Y.; Shah, K.J. Treatment of oil-contaminated water by modified polysilicate aluminum ferric sulfate. Processes 2018, 6, 95. [CrossRef]

2. Zhu, Z.; Xu, Y.; Luo, Y.; Wang, W.; Chen, X. Porous evaporators with special wettability for low-grade heat-driven water desalination. J. Mater. Chem. A 2020, 9, 702-726. [CrossRef] 
3. Mondal, S.; Bairi, P.; Das, S.; Nandi, A.K. Phase selective organogel from an imine based gelator for use in oil spill recovery. J. Mater. Chem. A 2019, 7, 381-392. [CrossRef]

4. Munirasu, S.; Haija, M.A.; Banat, F. Use of membrane technology for oil field and refinery produced water treatment-A review. Process. Saf. Environ. Prot. 2016, 100, 183-202. [CrossRef]

5. Yousef, R.; Qiblawey, H.; El-Naas, M.H. Adsorption as a process for produced water treatment: A review. Processes 2020, 8, 1657. [CrossRef]

6. Kweinor Tetteh, E.; Rathilal, S. Evaluating pre- and post-coagulation configuration of dissolved air flotation using response surface methodology. Processes 2020, 8, 383. [CrossRef]

7. Feng, L.; Li, S.; Li, Y.; Li, H.; Zhang, L.; Zhai, J.; Song, Y.; Liu, B.; Jiang, L.; Zhu, D. Super-hydrophobic surfaces: From natural to artificial. Adv. Mater. 2002, 14, 1857-1860. [CrossRef]

8. Zhu, Z.; Zhong, L.; Horseman, T.; Liu, Z.; Zeng, G.; Li, Z.; Lin, S.; Wang, W. Superhydrophobic-omniphobic membrane with anti-deformable pores for membrane distillation with excellent wetting resistance. J. Membr. Sci. 2021, 620, 118768. [CrossRef]

9. Wen, Q.; Di, J.; Jiang, L.; Yu, J.; Xu, R. Zeolite-coated mesh film for efficient oil-water separation. Chem. Sci. 2013, 4, 591-595. [CrossRef]

10. Xu, Y.; Tang, C.; Ma, J.; Liu, D.; Qi, D.; You, S.; Cui, F.; Wei, Y.; Wang, W. Low-tortuosity water microchannels boosting energy utilization for high water flux solar distillation. Environ. Sci. Technol. 2020, 54, 5150-5158. [CrossRef] [PubMed]

11. Liu, M.; Wang, S.; Wei, Z.; Song, Y.; Jiang, L. Bioinspired design of a superoleophobic and low adhesive water/solid interface. Adv. Mater. 2009, 21, 665-669. [CrossRef]

12. Cheng, Q.; Ye, D.; Chang, C.; Zhang, L. Facile fabrication of superhydrophilic membranes consisted of fibrous tunicate cellulose nanocrystals for highly efficient oil/water separation. J. Membr. Sci. 2017, 525, 1-8. [CrossRef]

13. Kim, S.; Cho, H.; Hwang, W. Robust superhydrophilic depth filter and oil/water separation device with pressure control system for continuous oily water treatment on a large scale. Sep. Purif. Technol. 2021, 256, 117779. [CrossRef]

14. Li, J.; Kang, R.; Tang, X.; She, H.; Yang, Y.; Zha, F. Superhydrophobic meshes that can repel hot water and strong corrosive liquids used for efficient gravity-driven oil/water separation. Nanoscale 2016, 8, 7638-7645. [CrossRef]

15. Lv, D.; Ou, J.; Hu, W.; Luo, X.; Wang, F. Superhydrophobic surface on copper via a one-step solvent-free process and its application in oil spill collection. RSC Adv. 2015, 5, 49459-49465. [CrossRef]

16. Cheng, Y.; Lu, S.; Xu, W.; Wen, H.; Wang, J. Fabrication of superhydrophobic Au-Zn alloy surface on a zinc substrate for roll-down, self-cleaning and anti-corrosion properties. J. Mater. Chem. A 2015, 3, 16774-16784. [CrossRef]

17. Zang, D.; Wu, C.; Zhu, R.; Zhang, W.; Yu, X.; Zhang, Y. Porous copper surfaces with improved superhydrophobicity under oil and their application in oil separation and capture from water. Chem. Commun. 2013, 49, 8410-8412. [CrossRef]

18. Liu, Y.; Ai, K.; Lu, L. Polydopamine and its derivative materials: Synthesis and promising applications in energy, environmental, and biomedical fields. Chem. Rev. 2014, 114, 5057-5115. [CrossRef]

19. Ryu, J.H.; Messersmith, P.B.; Lee, H. Polydopamine surface chemistry: A decade of discovery. ACS Appl. Mater. Interfaces 2018, 10, 7523-7540. [CrossRef]

20. Cao, Y.; Liu, N.; Zhang, W.; Feng, L.; Wei, Y. One-step coating toward multifunctional applications: Oil/water mixtures and emulsions separation and contaminants adsorption. ACS Appl. Mater. Interfaces 2016, 8, 3333-3339. [CrossRef]

21. Fang, W.; Liu, L.; Li, T.; Dang, Z.; Qiao, C.; Xu, J.; Wang, Y. Electrospun N-substituted polyurethane membranes with self-healing ability for self-cleaning and oil/water separation. Chem. Eur. J. 2016, 22, 878-883. [CrossRef] [PubMed]

22. Zhang, C.; Ou, Y.; Lei, W.X.; Wan, L.S.; Ji, J.; Xu, Z.K. CuSO ${ }_{4} / \mathrm{H}_{2} \mathrm{O}_{2}$-induced rapid deposition of polydopamine coatings with high uniformity and enhanced stability. Angew. Chem. Int. Ed. 2016, 55, 3054-3057. [CrossRef] [PubMed]

23. Chen, Y.; Wu, T.; Gao, P.; Li, N.; Wan, X.; Wang, J.; Pan, W.; Tang, B. A Cu ${ }^{2+}$ doped mesoporous polydopamine Fenton nanoplatform for low-temperature photothermal therapy. Mater. Chem. Front. 2021, 5, 6546-6552. [CrossRef]

24. Li, Q.; Zhang, T.; Chen, J.; Ji, W.; Wei, Y. In situ synthesis of fluorescent polydopamine polymer dots based on Fenton reaction for multi-sensing platform. J. Mate. Chem. B 2021, 9, 5503-5513. [CrossRef]

25. Zhu, Z.; Tan, G.; Lei, D.; Yang, Q.; Tan, X.; Liang, N. Omniphobic membrane with process optimization for advancing flux and durability toward concentrating reverse-osmosis concentrated seawater with membrane distillation. J. Membr. Sci. 2021, 639, 119763. [CrossRef] 